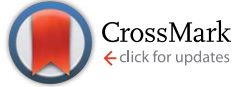

Cite this: RSC Adv., 2015, 5, 44408

\title{
Building cell-containing multilayered phospholipid polymer hydrogels for controlling the diffusion of a bioactive reagent $\dagger$
}

\begin{abstract}
Botao Gao, ${ }^{a}$ Tomohiro Konno ${ }^{b}$ and Kazuhiko Ishihara*ab
Cytocompatible and multilayered phospholipid polymer hydrogels containing living cells and a specific bioactive reagent were used to determine the role of diffusion of a bioactive reagent in regulating cellfate. Within the multilayered hydrogels prepared by a layer-by-layer assembling process, a bioactive reagent reservoir layer and a cell-laden layer were separated with a finely formed dual-crosslinked hydrogel multilayer that was composed of a 2-methacryloyloxyethyl phosphorylcholine polymer and photo-reactive poly(vinyl alcohol). The dual-crosslinked hydrogel was less permeable than the singlecrosslinked hydrogel and thus served as a diffusion-controlling barrier. We demonstrated the use of this multilayered hydrogel by subjecting the human cervical cancer HeLa cell-line to paclitaxel. We found that cell viability was regulated by the thickness of the diffusion-controlling barrier and the bioactive reagent concentration. An increase in the barrier thickness and a decrease in the bioactive reagent concentration resulted in decreased apoptosis of the HeLa cells. Our results suggest that the gradient of a bioactive reagent formed was due to the diffusion-controlling barriers, which explains the observed diffusion-dependent cell behavior. This study provides insight into the regulation of bioactive reagent diffusion in micrometer scale hydrogels and the associated diffusion-dependent effects on cell behavior. This finding can contribute to the design of a platform for studying diffusion-dependent cell behavior for tissue engineering and regenerative medicine applications.
\end{abstract}

Received 25th March 2015 Accepted 24th April 2015

DOI: $10.1039 / c 5 r a 05299 h$

www.rsc.org/advances

\section{Introduction}

Hydrogel matrices contain a large amount of water, therefore, they can provide viscoelastic mechanical properties similar to many soft tissues and permit diffusion of water as well as nutrients, proteins and signaling molecules. ${ }^{\mathbf{1 - 3}}$ Altogether, they mimic some key features of the native extracellular matrix (ECM) and facilitated cell spreading, migration and differentiation. ${ }^{4,5}$ Diffusion of bioactive reagents plays an important role in gradient formation within three-dimensional (3D) matrices, which are involved in the regulation of cell behavior. ${ }^{6-8}$ Mimicry of these gradients in hydrogels could be particularly important in functional tissue engineering approaches, which aim to use combinations of cells, materials, and bioactive reagents to recreate natural tissue structure and function. ${ }^{1}$

In recent years, there have been increasing studies that try to implement soluble or covalently-attached gradients of growth

${ }^{a}$ Department of Materials Engineering, The University of Tokyo, 7-3-1, Hongo, Bunkyo-ku, Tokyo 113-8656, Japan. E-mail: ishihara@mpc.t.u-tokyo.ac.jp; Fax: +813-5841-8647; Tel: +81-3-5841-7124

${ }^{b}$ Department of Bioengineering, School of Engineering, The University of Tokyo, 7-3-1, Hongo, Bunkyo-ku, Tokyo 113-8656, Japan

$\dagger$ Electronic supplementary information (ESI) available. See DOI: 10.1039/c5ra05299h factors, cytokines or adhesion sequences into 3D hydrogel matrices in order to control cell behavior such as migration, proliferation, apoptosis and differentiation. ${ }^{6,9-14}$ Several approaches such as photolithographic technology, ${ }^{\mathbf{1 0} 11}$ soft lithographic processes, ${ }^{12}$ and microfluidic technology, ${ }^{13,14}$ have been used to spatially control the diffusional properties of hydrogels. While these new approaches represent great progress, each one has its specific drawbacks, such as complicated manipulations or lengthy constructions.

Layer-by-layer (LbL) assembly technology, which can mimic the highly organized and stratified architectures of biological tissues, can be useful for the integration of specific reagents into biomaterials. Several studies using this technology have been carried out. ${ }^{15-17}$ It is an economical, easily applicable, and versatile tool for the controlled fabrication of surface coatings or tissue scaffolds.

A variety of bioactive reagents, such as proteins, DNA, RNA, and nanoparticles, can be incorporated into single layers to functionalize multilayered assembly. ${ }^{11-13}$ However, until recently, stable incorporation of cells into single layers of the LbL assembly was a challenge, with the exception of a cell spraying LbL procedure. ${ }^{18}$ For the first time, we have reported a spinning-assisted LbL procedure that allows precise spatial control of multiple cell types in 3D without cellular damage. ${ }^{19}$ This method has been previously used to encapsulate tumor 
cells and stroma cells to study distance-dependent cell-cell interactions. ${ }^{20}$

When a water-soluble phospholipid polymer, poly[2methacryloyloxyethyl phosphorylcholine (MPC)-co- $n$-butyl methacrylate (BMA)-co-p-vinylphenylboronic acid (VPBA)] (PMBV) and poly(vinyl alcohol) (PVA) were mixed together, based on the specific reaction between the VPBA unit in PMBV and the diol group in PVA, a hydrogel (PMBV/PVA) was spontaneously formed at room temperature. This hydrogel formation occurs under mild reaction conditions and can effectively encapsulate biological components, such as bacteria, ${ }^{21}$ living cells, ${ }^{22,23}$ and bioactive reagents. ${ }^{24,25}$

In this study, photo-reactive PVA (azido unit pendant watersoluble photopolymer, AWP ${ }^{26}$ was used to prepare a dualcrosslinked hydrogel (PMBV/AWP) to increase the crosslinking degree of the hydrogel. Thus, this hydrogel plays the role of a diffusion-controlling barrier, by which the diffusion rate of the bioactive reagents contained in the hydrogel can be controlled as a function of the thickness of the barrier layer. We used the anticancer bioactive reagent, paclitaxel (PTX), as a model bioactive reagent. Although PTX is extremely hydrophobic, it can be dissolved in a PMBV aqueous solution with high efficiency due to the presence of hydrophobic domains in aqueous medium. ${ }^{\mathbf{2 4 2 5}}$ Therefore, PTX can be loaded in situ into the PMBV/PVA hydrogel to prepare a reservoir layer of the bioactive reagent. What's more, we developed a spinningassisted LbL method for building a sandwich multilayered hydrogel platform, that is (PMBV/PVA as a PTX-loaded layer)(PMBV/AWP as a diffusion-controlling barrier)-(PMBV/PVA as a cell-incorporated layer). ${ }^{\mathbf{1 9} 20}$

The aim of this study was to investigate the diffusion of a bioactive reagent in sandwich structured multilayered hydrogels, and to determine the effect of the thickness of the diffusion-controlling barrier and the concentration of the bioactive reagent on cell behavior. The proposed methodology may contribute to the technological advancement of tissue engineering by offering the possibility to regulate the biomaterial composition and control different biological effects during tissue reconstruction.

\section{Experimental section}

\section{Materials}

MPC was synthesized by a previously reported method ${ }^{27}$ and purchased from NOF Co. Ltd. (Tokyo, Japan). BMA was purchased from Kanto Chemicals (Tokyo, Japan); VPBA, PVA (mean polymerization degree $=1.0 \times 10^{3}$ ), and PTX were purchased from Wako Pure Chemical Industries, Ltd. (Osaka, Japan). Methacryloxyethyl thiocarbonyl rhodamine B (MTR) was purchased from Polysciences (PA, USA). Fluorescence-labeled PTX (Oregon-green 488-labeled) was purchased from Invitrogen (Carlsbad, CA, USA). AWP was purchased from Tokyo Gosei Co. Ltd., Japan. Other organic reagents and solvents were commercially available reagents of extra-pure grade and were used without further purification.

PMBV was synthesized by a conventional free radical polymerization of corresponding monomers, as previously described. ${ }^{19,20}$ The mole fractions of MPC, BMA, and VPBA units in the obtained PMBV were determined by ${ }^{1} \mathrm{H}-\mathrm{NMR}$ (JNM-NR30; JEOL, Tokyo, Japan) and are 0.60, 0.25, and 0.15, respectively. The average molecular weight of PMBV was $1.7 \times 10^{4}$, as determined by a gel permeation chromatographic system (Jasco, Tokyo, Japan) with poly(ethylene oxide) standards. Fluorescence-labeled PMBV, poly(MPC-co-BMA-co-VPBA-co-MTR) (PMBV-R) was synthesized by radical polymerization. ${ }^{19}$ The chemical structures of PMBV and AWP are shown in Fig. 1a. The dual-crosslinking reaction between PMBV and AWP is shown in Fig. $1 \mathrm{~b}$.

\section{Cell culture procedure}

We used human cervical cancer HeLa cells that showed a stable expression of fluorescent ubiquitination-based cell cycle indicator (Fucci) fusion proteins. The Fucci fusion proteins were used as cell cycle sensors to perform quantitative analysis of cell activity kinetics. ${ }^{28}$ HeLa-Fucci cells were purchased from Riken Cell Bank (Ibaraki, Japan). They were cultured with DMEM (Sigma-Aldrich, St. Louis, MO) supplemented with $10 \%$ fetal bovine serum at $37^{\circ} \mathrm{C}$ in a $5.0 \mathrm{vol} \% \mathrm{CO}_{2}$ humidified atmosphere.

\section{Solubilization of PTX with the polymer solutions}

PTX was dissolved in a PMBV solution. ${ }^{24}$ Briefly, PTX $1.0 \mathrm{mg}$ $\mathrm{mL}^{-1}$ ) was dissolved in $99.5 \mathrm{wt} \%$ ethanol, and PMBV was dissolved in PBS with polymer concentrations of $50 \mathrm{mg} \mathrm{mL}^{-1}$. Then a PTX-loaded PMBV solution was prepared by vortexing a mixture of the PTX and PMBV solutions at a ratio of PMBV/ PTX $=90 / 10$, and then removing the ethanol under reduced pressure. The PTX that was not dissolved was removed by filtration. The final PTX concentration in the PMBV solution was $75 \mu \mathrm{M}$ (data not shown).

\section{Preparation of dual-crosslinked PMBV/AWP multilayer hydrogels}

The PMBV(-R) solution (5.0 wt $\%$ ) was prepared in PBS. The AWP solution (2.0 wt\%) was prepared using a diluted stock solution (6.0 wt $\%$ ) comprised of a mixture of $99.5 \mathrm{wt} \%$ ethanol and PBS at a ratio of $\mathrm{PBS} /$ ethanol $=30 / 10$. The $\mathrm{PMBV} / \mathrm{AWP}$ multilayer hydrogel was prepared by a spinning assisted LbL procedure (Fig. 2) similar to our previous report. ${ }^{15,16}$ Briefly, the AWP solution was manually spread on a $35 \mathrm{~mm}$ plastic culture dish to form a thin membrane. AWP-coated dishes were then air-dried in a fume hood at room temperature. Then they were irradiated for $1.0 \mathrm{~min}$ with UV light $\left(50 \mathrm{~mW} \mathrm{~cm}^{-2}\right)$ from a UV Spot Cure (SP-7, Ushio Inc., Yokohama, Japan). Then the PMBV solution was deposited onto the AWP coated surface. After $1.0 \mathrm{~min}$, to allow the boronic acid-diol interaction, the dish was rotated at $2000 \mathrm{rpm}$ to remove the excess PMBV solution and form a layer of PMBV/AWP membrane. Next, the AWP solution and PMBV solutions were alternately deposited to form a (PMBV/AWP)$(\mathrm{PMBV} / \mathrm{AWP})_{n}$ hydrogel membrane, where the value of $n(n=1$, $3,5)$ indicates the number of PMBV/AWP layers. Finally, the multilayered hydrogel membranes were air-dried, irradiated with UV light $\left(50 \mathrm{~mW} \mathrm{~cm}^{-2}\right)$ for $3.0 \mathrm{~min}$ and submerged in PBS. With the help of PMBV-R to indicate the PMBV distribution in the multilayered hydrogels, the structure and thickness of the 
(a)

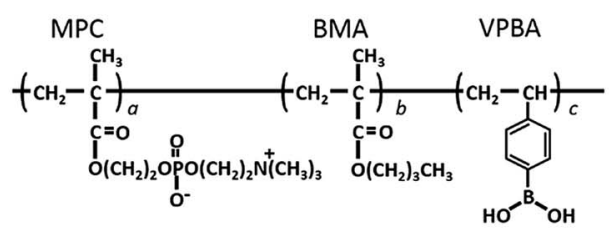

$\operatorname{PMBV}(\mathrm{a} / \mathrm{b} / \mathrm{c}=0.6 / 0.25 / 0.15)$<smiles>CC(C)CC(O)CC(O)CC(O)CC(O)CC1CC(CC(C)C)C(C(=O)NC(=O)C=Cc2ccc(N)cc2)C(C)O1</smiles>

Photo-reactive polyvinylalcohol (Azido unit pendant Water-soluble Photopolymer, AWP)

(b) Chemical- and photo-crosslinking mechanism
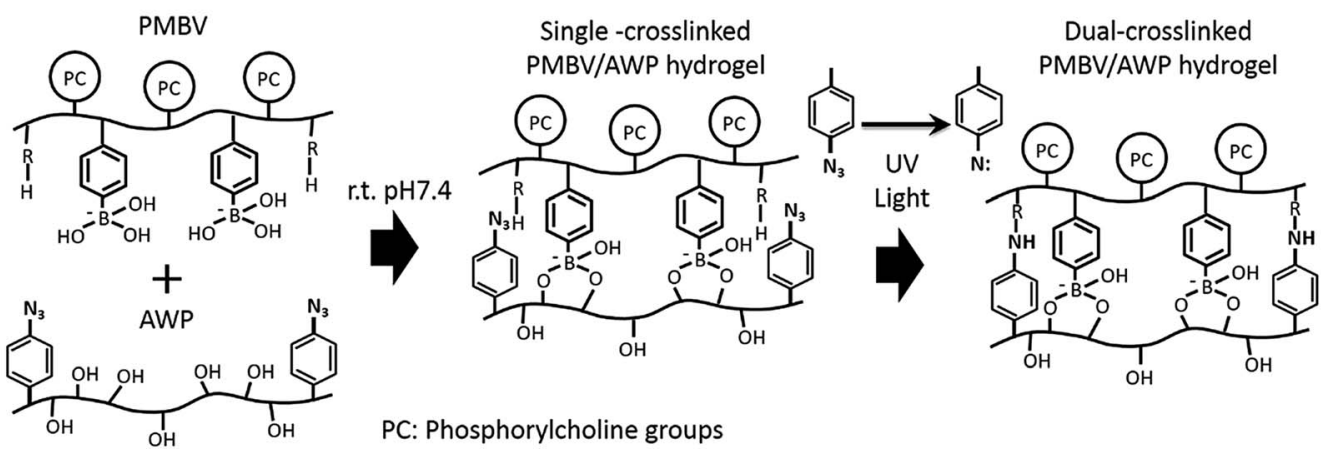

Fig. 1 (a) The chemical structure of PMBV and AWP, and (b) the dual-crosslinking mechanism of the PMBV/AWP hydrogel.

multilayered hydrogels were characterized using a Zeiss LSM 510 confocal laser-scanning microscope (CLSM; Carl Zeiss, Oberkochen, Germany).

For the sample preparation for FT-IR reflection absorption spectroscopy (RAS), the dual-crosslinked multilayered hydrogels were applied onto Au spattered glass substrates and irradiated with UV light for 0 or $3.0 \mathrm{~min}$. After drying overnight in a desiccator, the membranes were measured by FT-IR RAS (FT-IR 6300/Jasco).
Incorporation of a bioactive reagent and cells into the multilayered hydrogels

The following procedure is shown in Fig. 2. HeLa-Fucci cells were dispersed in 5.0 wt\% PMBV solution, and a $5.0 \mathrm{wt} \%$ PMBV solution containing PTX (750 nM, $375 \mathrm{nM}$, and $188 \mathrm{nM}$ ) was also prepared. Briefly, the $5.0 \mathrm{wt} \%$ PVA solution was dropped on an irradiated PMBV/AWP layer and spun at 2000 rpm followed by the deposition of $50 \mu \mathrm{L}$ PMBV solution

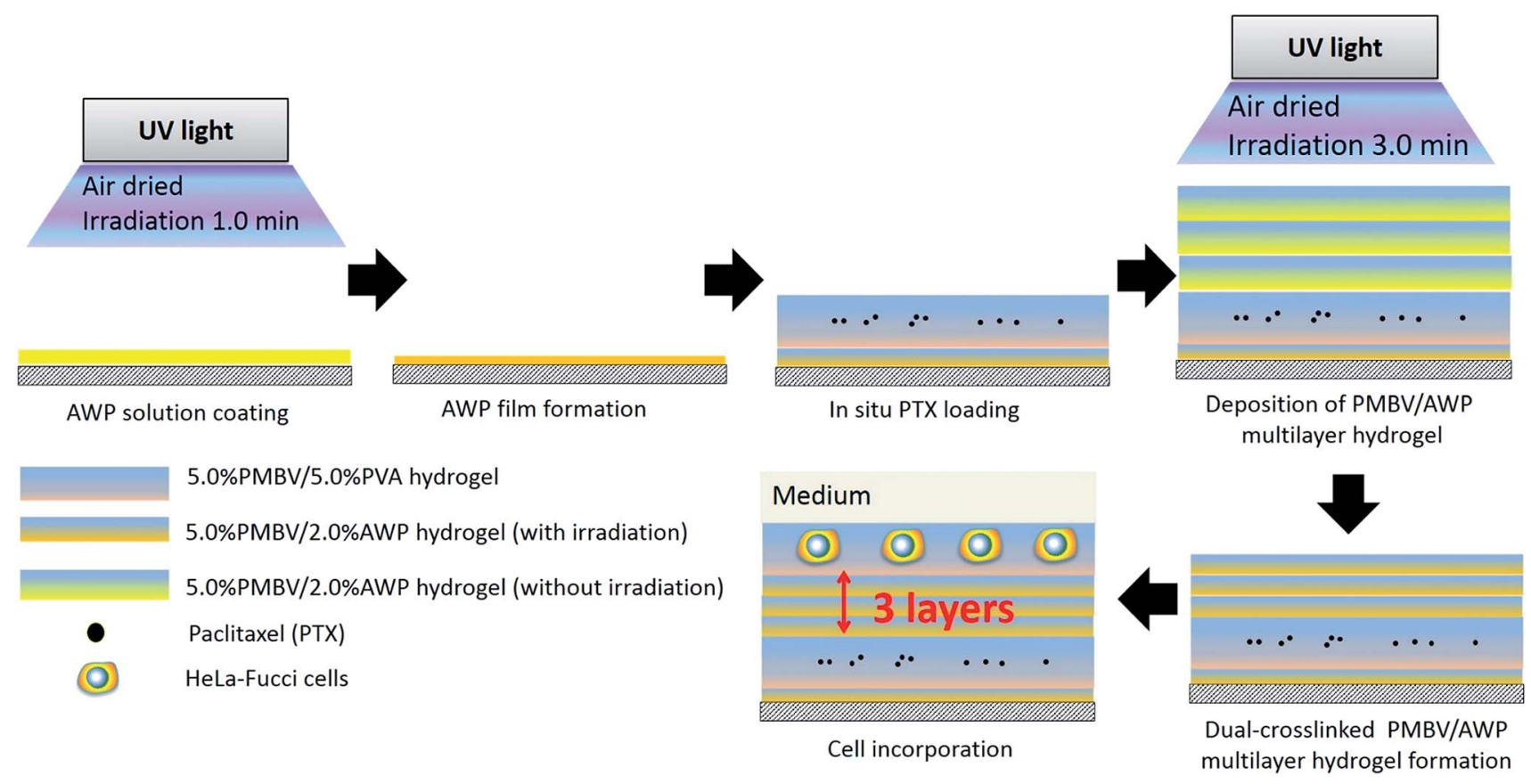

Fig. 2 Fabrication of the multilayered hydrogel with encapsulation of PTX and cells. 
Table 1 Experimental groups to study HeLa-Fucci cells associated with PTX stimulation under different conditions

\begin{tabular}{llr}
\hline Experimental group & Number of hydrogel layers ${ }^{a}$ & Concentration of PTX \\
\hline Thick barrier & $5^{b}$ & $375 \mathrm{nM}$ \\
Intermediate barrier & $3^{b}$ & $375 \mathrm{nM}$ \\
Thin barrier & $1^{b}$ & $375 \mathrm{nM}$ \\
Control & $5^{c}$ & $375 \mathrm{nM}$ \\
High concentration & $1^{b}$ & $750 \mathrm{nM}$ \\
Intermediate concentration & $1^{b}$ & $375 \mathrm{nM}$ \\
Low concentration & $1^{b}$ & $188 \mathrm{nM}$
\end{tabular}

${ }^{a}$ Number of hydrogel layers between the PTX-loaded layer and the HeLa-Fucci cell laden layer. ${ }^{b}$ Number of PMBV/AWP hydrogel layers (diffusioncontrolling barrier). ${ }^{c}$ Number of PMBV/PVA hydrogel layers.

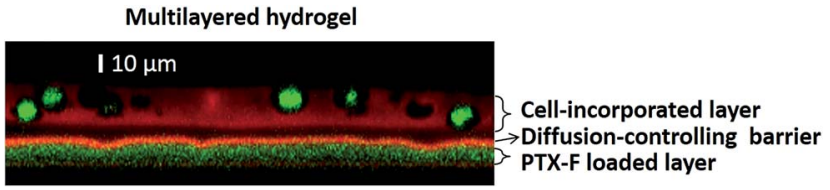

Fig. 3 Confocal microscopy observation of the structure of a multilayered hydrogel with the encapsulation of PTX and cells: (PMBV-R/ AWP)-(PMBV/PTX-F/PVA)-(PMBV-R/AWP) ${ }_{3}-(\mathrm{PMBV}-\mathrm{R} / \mathrm{HeLa}-\mathrm{FucC}$ cell/PVA)-(PMBV/PVA) (scale bars: $10 \mu \mathrm{m})$.

containing PTX. Then the PTX loaded layer was alternately deposited with AWP solution and PMBV solution to form a (PMBV/AWP)-(PMBV/PTX/PVA)-(PMBV/AWP $)_{n}$ multilayer hydrogel, where the value of $n=1,3$, and 5 . Next, $4.0 \mu \mathrm{L}$ PMBV/HeLa-Fucci cell suspension was spotted on the top of the growing multilayered hydrogel. The final structure was denoted as a (PMBV/AWP)-(PMBV/PTX/PVA)-(PMBV/AWP $)_{n}-$ (PMBV/HeLa-Fucci cell/PVA)-(PMBV/PVA) multilayer hydrogel. Finally, 2.0 mL DMEM was added to each dish and all the samples were incubated at $37{ }^{\circ} \mathrm{C}$ in a 5.0 vol\% $\mathrm{CO}_{2}$ humidified atmosphere for $48 \mathrm{~h}$.

We used four groups to evaluate the cell response as a function of the diffusion-controlling barrier thickness, which are shown in Table 1: a thick barrier group (5 layers PMBV/ AWP), an intermediate barrier group (3 layers PMBV/AWP), a thin barrier group (1 layer PMBV/AWP), and a control group (5 layers PMBV/PVA).
Another three groups were used to evaluate the cell response as a function of the bioactive reagent concentration, with a constant barrier thickness (1 layer PMBV/AWP), including a high concentration group (750 nM PTX), an intermediate concentration group (375 nM PTX), and a low concentration group (188 nM PTX).

\section{Evaluation of the cell proliferation-cycle distribution and cell viability}

The numbers, positions, and fluorescence of HeLa-Fucci cells were measured using a BIOREVO BZ-9000 fluorescence microscope (Keyence, Osaka, Japan) at $12 \mathrm{~h}$ and $48 \mathrm{~h}$. For quantitative analysis of fluorescent cells, at least 300 cells in five independent fields were analyzed.

Cells designated as apoptotic were those that displayed the characteristic morphological features of apoptosis, including cell volume shrinkage, chromatin condensation, and the presence of membrane-bound apoptotic bodies. According to this apoptotic cell morphology, the percentage of apoptotic cells was calculated.

\section{Evaluation of bioactive reagent diffusivity in dual-crosslinked PMBV/AWP multilayer hydrogels}

For the evaluation of bioactive reagent diffusion in the PMBV/ AWP hydrogels, the diffusion coefficient of PTX-F was determined by fluorescence correlation spectroscopy (FCS) measurements (CLSM, Carl Zeiss) as previously described. ${ }^{29}$

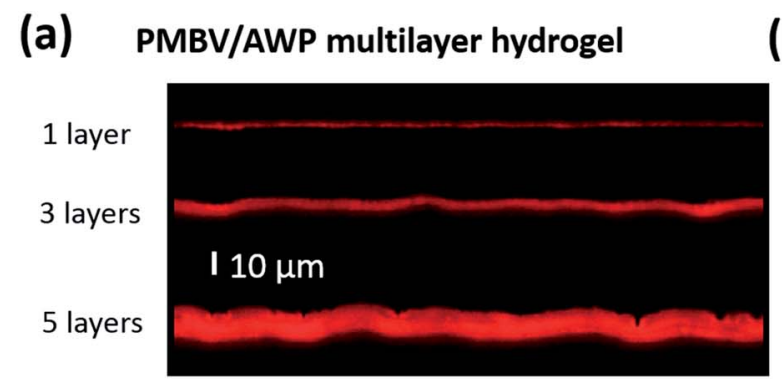

(b)

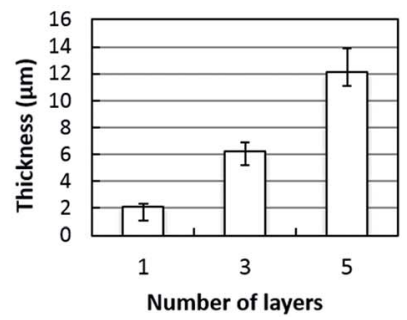

Fig. 4 Thickness regulation of the PMBV/AWP multilayer hydrogel. (a) Confocal Z section of the dual-crosslinked PMBV-R/AWP multilayer hydrogel and $(b)$ the thickness of the PMBV/AWP multilayer hydrogels exhibit a linear growth behavior with the increase in the number of layers (scale bars: $10 \mu \mathrm{m}$ ). 
(a)
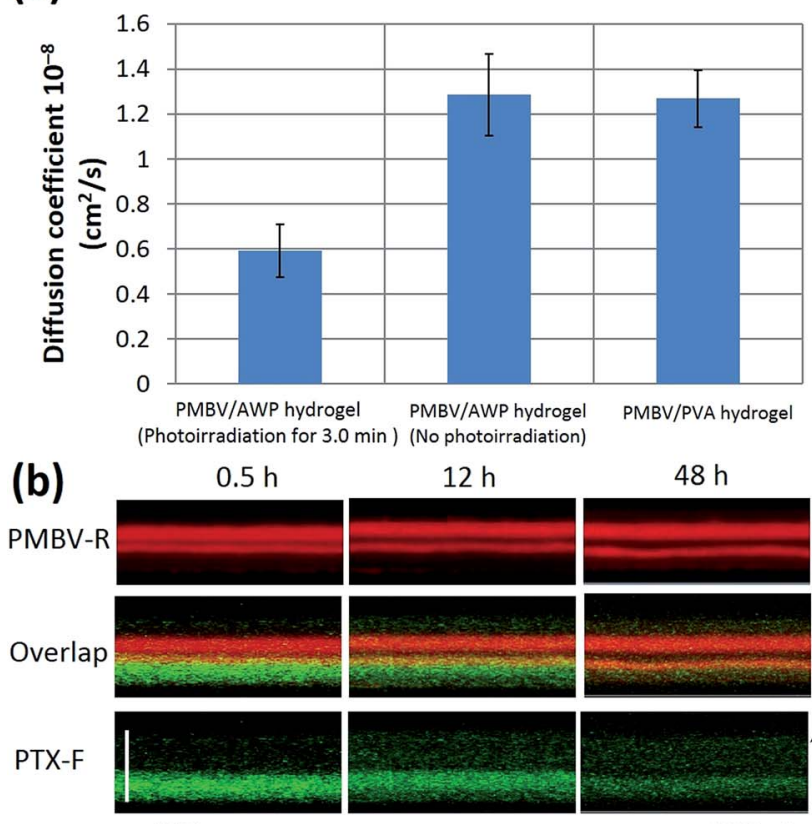

$12 \mathrm{~h}$
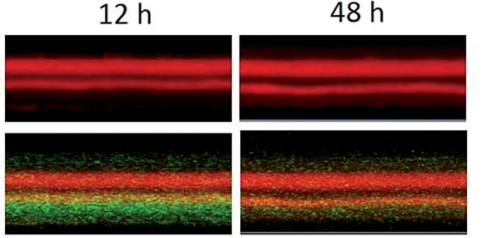

(c)

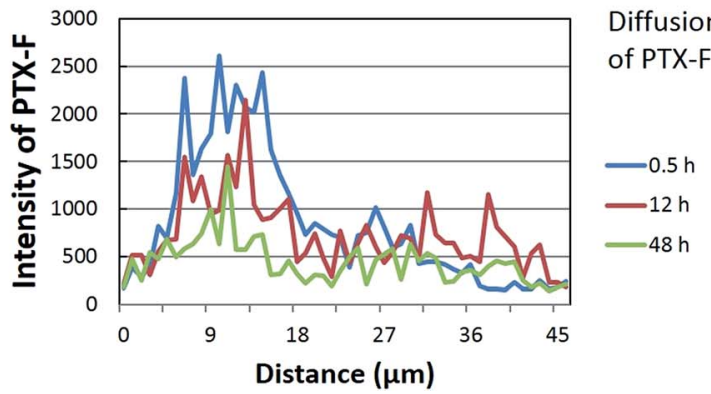

Fig. 5 Bioactive reagent diffusivity within multilayered hydrogels (a) The diffusion coefficients of PTX-F determined within multilayered hydrogels. (b) Time lapse images of a functionalized multilayer hydrogel dosed with $2 \mu \mathrm{M}$ fluorescence labeled PTX (PTX-F) in the bottom layer. (c) Fluorescent intensities were obtained starting from the bottom of the gel and to a distance of $45 \mu \mathrm{m}$ from the bottom for three different time points. The fluorescent intensity of PTX-F was plotted as a function of distance from the bottom (scale bars: $45 \mu \mathrm{m})$.

The PTX-F/PBS solution (200 nM, $2.0 \mathrm{~mL}$ ) was added onto multilayered PMBV/AWP (0 min irradiation), multilayered PMBV/AWP (3.0 min irradiation), and multilayered PMBV/ PVA, which were built on $35 \mathrm{~mm}$ (glass 27ф) glass base dishes (Iwaki) and stored at $37{ }^{\circ} \mathrm{C}$ for $24 \mathrm{~h}$ to equilibrate the samples. Then the diffusion coefficients of PTX-F were determined at eight positions within the multilayered hydrogels to get an average value. The experiments were repeated three times.

In order to observe the diffusion process of PTX-F within the multilayered hydrogel, a (PMBV-R/AWP)-(PMBV/2.0 $\mu \mathrm{M}$ PTX-F/ PVA)-(PMBV-R/AWP) $)_{3}$ (PMBV-R/PVA) (PMBV/PVA) multilayer hydrogel was prepared. Cross-section fluorescence images of the multilayered hydrogel were taken at $0.5,12$, and $48 \mathrm{~h}$ under the same settings using a confocal laser-scanning microscope (Carl Zeiss).

\section{Statistical analysis of the data}

A student's $t$-test was carried out to determine whether the observed differences were statistically significant $(p<0.05)$.

\section{Results and discussion}

\section{Fabrication of multifunctional hydrogel layers}

The LbL assembling procedure was applied for the preparation of multilayered hydrogels composed of PMBV and PVA. We successfully controlled the thickness of the multilayered hydrogels by changing both the polymer concentration and the number of layers. ${ }^{19}$

In this study, we embedded PTX in a PMBV/PVA layer, and then separated this layer from a cell-containing PMBV/PVA layer by PMBV/AWP layers. These multilayered hydrogels are novel because: (1) their micrometer size allows for the incorporation of both bioactive reagents and cells in their architecture, which would not be easily achieved by other LbL technologies, and (2) separating the bioactive reagent-loaded layer and the cellcontaining layer with different thicknesses of PMBV/AWP layers in a multilayered hydrogel allows for fine-tuned accessibility of bioactive reagents into the cells.

As shown in Fig. 3, confocal microscopy imaging shows a multilayered hydrogel consisting of fluorescent-labeled PMBV, fluorescent-labeled PTX, and HeLa-Fucci cells. In the vertical cross-section, a sandwich structure of the bioactive reagent loaded layer, diffusion-controlling barrier, and cellincorporated layer was visualized. As shown in Fig. 4a and b, the thickness of dual-crosslinked PMBV/AWP could be finely tuned by varying the number of gel layers. The HeLa-Fucci cellladen PMBV/PVA layer was on top of the dual-crosslinked PMBV/AWP layers. The thickness of one layer of 5.0\%-PMBV/ $5.0 \%$-PVA was about $15-20 \mu \mathrm{m}$, which was enough to hold cells. Our previous research proved that the concentration of PVA was very important to form a cell-containing layer, because it could provide enough thickness and mechanical properties to hold the cells due to its relatively high viscosity. ${ }^{19}$

To confirm the photoreaction in the dual-crosslinked hydrogel membranes, dried membranes were measured by FT-IR RAS (see Fig. S1 in the ESI $\dagger$ ). A specific peak of the azide group was detected at $2123 \mathrm{~cm}^{-1}$ before UV irradiation. This peak was eliminated after UV irradiation, indicating a photochemical-crosslinking of the azide group.

\section{Bioactive reagent diffusivity within multilayered hydrogels}

To understand the permeability of the dual-crosslinked hydrogels, we measured the diffusion coefficient of PTX-F across different multilayered hydrogels. As shown in Fig. 5a, the diffusion coefficient of PTX-F across the PMBV/AWP hydrogel (photoirradiation for $3.0 \mathrm{~min}$ ) was much lower than the singlecrosslinked PMBV/AWP hydrogel (no irradiation) or PMBV/PVA. A probable reason for this phenomenon is that dual-crosslinked PMBV/AWP has the highest crosslinking degree, which prevents the diffusion of PTX-F. Therefore, PMBV/AWP could work as a diffusion-controlling barrier and generate unique spatial gradients of PTX-F. As shown in Fig. 5b, PTX-F was encapsulated 
(a)
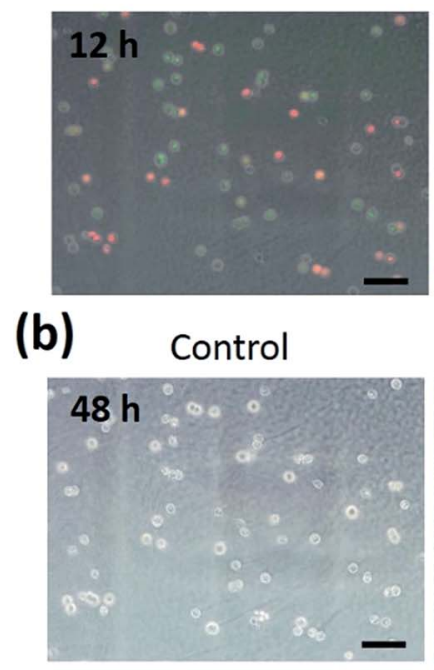

(c)

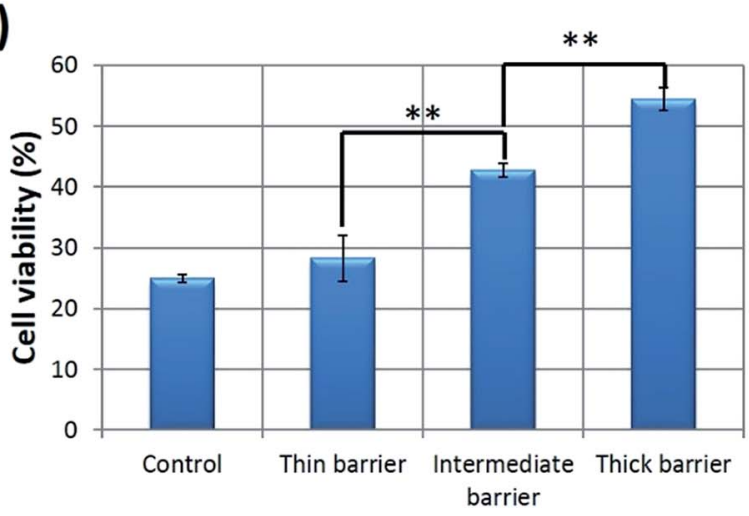

Experimental groups
Thin barrier

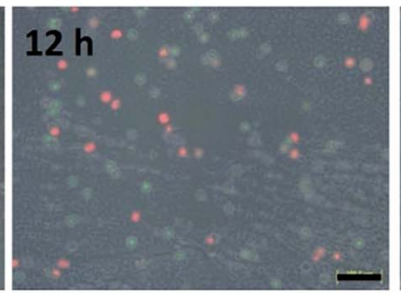

Thin barrier

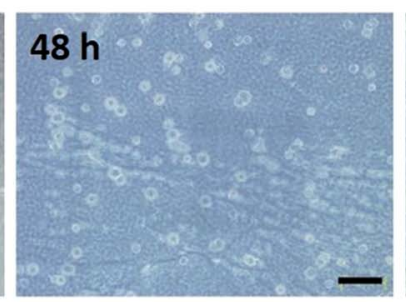

\section{(d)} shown as the average $\pm \operatorname{SD}(n=3, \alpha=0.05, * p<0.05)$ (scale bars: $100 \mu \mathrm{m})$.

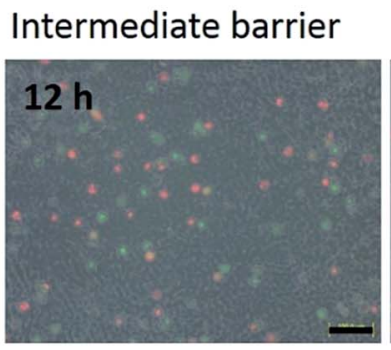

Intermediate barrier

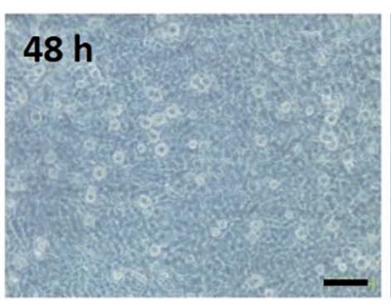

\section{Thick barrier}

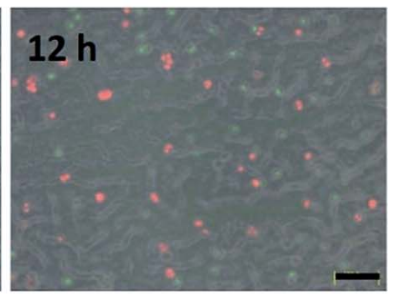

Thick barrier

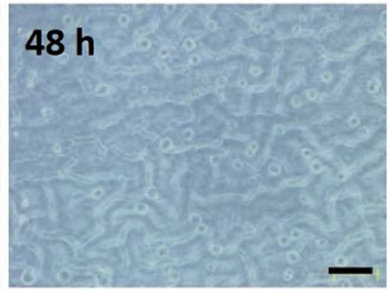

(d) $\boxminus$ Abnormal $\square \mathrm{S} / \mathrm{G} 2 / \mathrm{M} *$

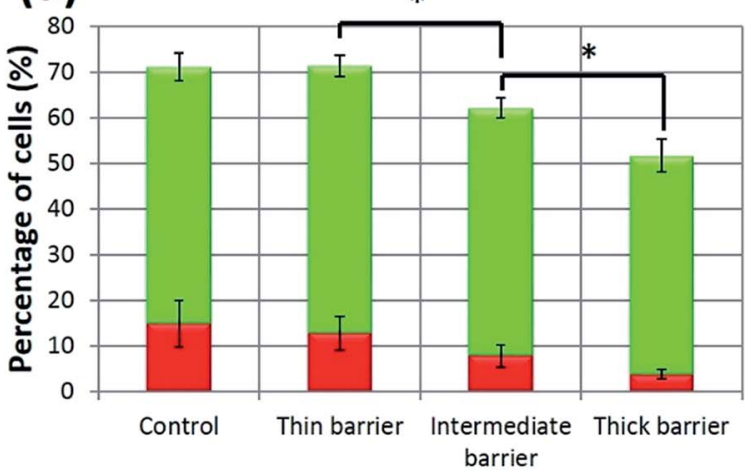

Experimental groups

Fig. 6 Regulation of cell cycle distribution and cellular viability by the thickness of the diffusion-controlling barrier. (a) Fluorescent images of HeLa-Fucci cells encapsulated within the top layer of a multilayer hydrogel is shown for the cell cycle distribution at $12 \mathrm{~h}$; (b) bright-field images show the viability of HeLa-Fucci cells at $48 \mathrm{~h}$; (c) cell viability is shown as the average $\pm \operatorname{SD}(n=3, \alpha=0.05, * * p<0.01)$; (d) cell cycle distribution,

into a reservoir, and was allowed to gradually diffuse through the diffusion-controlling barrier. Over the course of $48 \mathrm{~h}$, green fluorescent PTX-F gradually emanated, providing a spatial gradient field over a micrometer-size scale (Fig. $5 b$ and c). The evolution of the PTX-F intensity gradients demonstrated that the gradient gradually decreased with time and reached equilibrium after $48 \mathrm{~h}$ (Fig. 5c).

One point that should be emphasized here is the constant permeability of the PMBV/PVA (or AWP) hydrogel under cell culture conditions. As mentioned above, the PMBV/PVA hydrogel is a chemical crosslinked hydrogel. The boronic acid/diol complex can be dissociated by sugar solutions, such as sorbitol, which has a stronger affinity to boronic acids than that of the diol group, and thus degrades the PMBV/PVA hydrogel. ${ }^{23,30,31}$ There are no matrix metalloproteinase (MMP) sensitive segments in the PMBV/PVA hydrogel, so the PMBV/PVA hydrogel cannot be destroyed by MMPs secreted by cells. Therefore, it is impossible for the cells to degrade the hydrogels and subsequently enhance the permeability of the hydrogel.
Actually, it was found that the cells embedded in the PMBV/PVA hydrogel were rounded and they could not spread or migrate in the hydrogel. ${ }^{20,22,23,29-32}$

\section{Effects of PTX on the cell proliferation-cycle distribution}

Next, we demonstrated the applicability of our approach to control PTX diffusion within the hydrogel layers by changes in the thickness of the diffusion-controlling barrier. First, we determined the effects of PTX on the cell proliferation-cycle distribution in HeLa-Fucci cells at $12 \mathrm{~h}$ after stimulation. Fucci allows determination of the dynamics of live-cell proliferation during a cell cycle; G1 and S/G2/M phase cells expressing Fucci emit red and green fluorescence, respectively. ${ }^{28}$ At $12 \mathrm{~h}$, significant G2/M arrest was observed (Fig. 6a and d). Besides, confirming the previous report, ${ }^{33}$ we also observed that abnormal red fluorescence appeared in addition to the original green fluorescence at the $\mathrm{M}$ phase. Cells collapsed several hours after the appearance of the abnormal red fluorescence, 
suggesting that the emergence of the abnormal red fluorescence is an early indicator of mitotic catastrophe. As shown in Fig. 6d, the percentage of (G2/M phase cells + abnormal cells) in the four groups in descending order were as follows: control group $\approx$ thin barrier group $>$ intermediate barrier group $>$ thick barrier group. No significant differences were observed between the control group and the thin barrier group. This shows that one can tune the biological activity by altering the number of PMBV/ AWP layers composing the barrier. In order to further verify this barrier thickness dependent phenomenon, the cell viability of the above four groups was also characterized. Fig. $6 \mathrm{~b}$ and $\mathrm{c}$ show the cell viability after $48 \mathrm{~h}$ of bioactive reagent stimulation. Indeed, there were significant differences among them. While one PMBV/AWP layer does not show a significant barrier effect compared with the control group, five layers efficiently hinder the effects of PTX. A barrier composed of three PMBV/AWP layers partially hinders the diffusion of PTX, leading to a cellular viability between that of one and five PMBV/AWP layers. In addition, we found a dose effect: higher embedded-PTX concentrations lead to higher apoptosis of HeLa-Fucci cells (see Fig. S2 in the ESI $\dagger$ ).

These results can be explained based on the following mechanism: PTX can diffuse through a diffusion-controlling barrier and into the cell-containing gel and can come in contact with the cells. However, the amount of PTX in the cellcontaining hydrogel is dependent on the thickness of the diffusion-controlling barrier and the initial concentration of the loaded bioactive reagent. With an increasing number of barrier layers, the diffusion of PTX became limited and induced a weaker stimulation effect to the HeLa-Fucci cells.

This strategy will now be extended to include the incorporation of customized multi-agents and cell-containing systems to mimic diffusion-controlled cell behavior in vivo. We envision that this system could serve as a powerful tool in tissue engineering and cell biology to address basic questions related to spatiotemporal signaling of biomolecules, such as morphogens. For example, the recapitulation of morphogen gradients within 3D structures and an examination of their influence on the fate of developing pluripotent stem cells, is possible. ${ }^{34,35}$

\section{Conclusion}

In this study, we have engineered sandwich structured multilayered hydrogels where a cell-containing gel layer was separated from the bioactive reagent reservoir layer by dualcrosslinked hydrogels. The multilayered hydrogels could be micropatterned three-dimensionally by a single mechanism or dual mechanisms to spatially control the hydrogel permeability on the micrometer scale and permit examination of the diffusion of the bioactive reagent on encapsulated cell behavior. By manipulating the thickness of the dual-crosslinked hydrogel, which worked as a diffusion-controlling barrier, the barrier effects were tunable. Thus, we have shown that PTX embedded in the reservoir layer decreased apoptosis in HeLa cells as the barrier thickness increased and the PTX concentration decreased. Overall, the permeability of the hydrogel system was introduced as a design parameter for regulating cellular behavior, and this multilayered hydrogel system provides a new platform for studying diffusion-dependent cell behavior for tissue engineering and regenerative medicine applications.

\section{Acknowledgements}

This work was supported by a Grant-in-Aid for Scientific Research on Innovative Areas "Nanomedicine Molecular Science" (no. 2306) from the Ministry of Education, Culture, Sports, Science and Technology of Japan. One of the authors (B.G.) would like to acknowledge the Chinese Scholarship Council (CSC) for financial support.

\section{References}

1 L. G. Griffith and M. A. Swartz, Nat. Rev. Mol. Cell Biol., 2006, 7, 211-224.

2 H. K. Lau and K. L. Kiick, Biomacromolecules, 2015, 16, 28-42.

3 M. K. Gupta, J. R. Martin, T. A. Werfel, T. Shen, J. M. Page and C. L. Duvall, J. Am. Chem. Soc., 2014, 136, 14896-14902.

4 H. J. Lee, A. Sen, S. Bae, J. S. Lee and K. Webb, Acta Biomater., 2015, 14, 43-52.

5 S. Das, F. Pati, Y. J. Choi, G. Rijal, J. H. Shim, S. W. Kim, A. R. Ray, D. W. Cho and S. Ghosh, Acta Biomater., 2015, 11, 233-246.

6 T. Lühmann and H. Hall, Materials, 2009, 2, 1058-1083.

7 C. Fan and D. A. Wang, Macromol. Biosci., 2015, 15, 535-545.

8 J. B. Gurdon and P. Y. Bourillot, Nature, 2001, 413, 797-803.

9 B. J. Peret and W. L. Murphy, Adv. Funct. Mater., 2008, 18, 3410-3417.

10 O. Jeon and E. Alsberg, Adv. Funct. Mater., 2013, 23, 47654775 .

11 K. A. Mosiewicz, L. Kolb, A. J. van der Vlies, M. M. Martino, P. S. Lienemann, J. A. Hubbell, M. Ehrbar and M. P. Lutolf, Nat. Mater., 2013, 12, 1072-1078.

12 G. Eng, B. W. Lee, H. Parsa, C. D. Chin, J. Schneider, G. Linkov, S. K. Sia and G. Vunjak-Novakovic, Proc. Natl. Acad. Sci. U. S. A., 2013, 110, 4551-4556.

13 M. P. Cuchiara, A. C. Allen, T. M. Chen, J. S. Miller and J. L. West, Biomaterials, 2010, 31, 5491-5497.

14 J. S. Miller, K. R. Stevens, M. T. Yang, B. M. Baker, D. H. Nguyen, D. M. Cohen, E. Toro, A. A. Chen, P. A. Galie, X. Yu, R. Chaturvedi, S. N. Bhatia and C. S. Chen, Nat. Mater., 2012, 11, 768-774.

15 P. T. Hammond, Mater. Today, 2012, 15, 196-206.

16 V. Gribova, R. Auzely-Velty and C. Picart, Chem. Mater., 2011, 24, 854-865.

17 A. Shukla and B. Almeida, Wiley Interdiscip. Rev.: Nanomed. Nanobiotechnol., 2014, 6, 411-421.

18 L. Grossin, D. Cortial, B. Saulnier, O. Felix, A. Chassepot, G. Decher, P. Netter, P. Schaaf, P. Gillet, D. Mainard, J. C. Voegel and N. Benkirane-Jessel, Adv. Mater., 2009, 21, 650-655.

19 B. Gao, T. Konno and K. Ishihara, Colloids Surf., B, 2013, 108, 345-351.

20 B. Gao, T. Konno and K. Ishihara, Biomaterials, 2014, 35, 2181-2187. 
21 X. Lin, K. Nishio, T. Konno and K. Ishihara, Biomaterials, 2012, 33, 8221-8227.

22 Y. Xu, K. Sato, K. Mawatari, T. Konno, K. Jang, K. Ishihara and T. Kitamori, Adv. Mater., 2010, 22, 3017-3021.

23 H. Oda, T. Konno and K. Ishihara, Biomaterials, 2013, 34, 5891-5896.

24 J. Choi, T. Konno, M. Takai and K. Ishihara, Biomaterials, 2009, 30, 5201-5208.

25 J. Choi, T. Konno, M. Takai and K. Ishihara, Biomaterials, 2012, 33, 954-961.

26 Y. Ito, M. Nogawa, M. Takeda and T. Shibuya, Biomaterials, 2005, 26, 211-216.

27 K. Ishihara, T. Ueda and N. Nakabayashi, Polym. J., 1990, 22, 355-360.

28 A. Sakaue-Sawano, H. Kurokawa, T. Morimura, A. Hanyu, H. Hama, H. Osawa, S. Kashiwagi, K. Fukami, T. Miyata,
H. Miyoshi, T. Imamura, M. Ogawa, H. Masai and A. Miyawaki, Cell, 2008, 132, 487-498.

29 T. Aikawa, T. Konno and K. Ishihara, Soft Matter, 2013, 9, 4628-4634.

30 B. Gao, T. Konno and K. Ishihara, Eur. Polym. J., 2015, DOI: 10.1016/j.eurpolymj.2015.03.030.

31 H. Oda, T. Konno and K. Ishihara, Biomaterials, 2015, 56, 8691.

32 T. Konno and K. Ishihara, Biomaterials, 2007, 28, 1770-1777. 33 A. Honda-Uezono, A. Kaida, Y. Michi, K. Harada, Y. Hayashi, Y. Hayashi and M. Miura, Biochem. Biophys. Res. Commun., 2012, 428, 224-229.

34 O. Lieleg and K. Ribbeck, Trends Cell Biol., 2011, 21, 543-551. 35 N. Gjorevski, A. Ranga and M. P. Lutolf, Development, 2014, 141, 1794-1804. 